\title{
Disentangling discrepancies between stellar evolution theory and sub-solar mass stars
}

\section{The influence of the mixing length parameter for the UV Psc binary}

\author{
E. Lastennet ${ }^{1}$, J. Fernandes ${ }^{1}$, D. Valls-Gabaud ${ }^{2}$, and E. Oblak ${ }^{3}$ \\ 1 Observatório Astronómico da Universidade de Coimbra, Santa Clara, 3040 Coimbra, Portugal \\ 2 UMR CNRS 5572, Laboratoire d'Astrophysique, Observatoire Midi-Pyrénées, 14 avenue Édouard Belin, \\ 31400 Toulouse, France \\ 3 UMR CNRS 6091, Laboratoire d'Astrophysique, Observatoire de Besançon, 41bis avenue de l'Observatoire, BP 1615, \\ 25010 Besançon, France
}

Received 23 July 2002 / Accepted 16 July 2003

\begin{abstract}
Serious discrepancies have recently been observed between predictions of stellar evolution models in the $0.7-1.1 M_{\odot}$ mass range and accurately measured properties of binary stars with components in this mass range. We study one of these objects, the eclipsing binary UV Piscium, which is particularly interesting because Popper (1997) derived age estimates for each component that differed by more than a factor of two. In an attempt to solve this significant discrepancy (a difference in age of $11 \mathrm{Gyr}$ ), we compute a large grid of stellar evolution models with the CESAM code for each component. By fixing the masses to their accurately determined values (relative error smaller than $1 \%$ for both stars), we consider a wide range of possible metallicities $Z$ ( 0.01 to 0.05 ), and helium content $Y$ ( 0.25 to 0.34$)$ uncorrelated to $Z$. In addition, the mixing length parameter

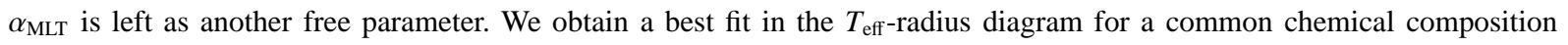
$(Z, Y)=(0.012,0.31)$, but a different MLT parameter $\alpha_{\text {MLT,A }}=0.95 \pm 0.12$ (statistical) +0.30 (systematic) and $\alpha_{\text {MLT,B }}=0.65 \pm$ 0.07 (stat) +0.10 (syst). The apparent age discrepancy found by Popper (1997) disappears with this solution, the components being coeval to within $1 \%$. This suggests that fixing $\alpha_{\mathrm{MLT}}$ to its solar value $(\sim 1.6)$, a common hypothesis assumed in most stellar evolutionary models, may not be correct. Secondly, since $\alpha_{\mathrm{MLT}}$ is smaller for the less massive component, this suggests that the $\alpha_{\text {MLT }}$ parameter may decrease with stellar mass, showing yet another shortcoming of the mixing length theory to explain stellar convection. This trend needs further confirmation with other binary stars with accurate data.
\end{abstract}

Key words. stars: individual: UV Psc - stars: fundamental parameters - stars: binaries: eclipsing - stars: abundances stars: Hertzsprung-Russell (HR) and C-M diagrams

\section{Introduction}

It is of the utmost importance for stellar evolution theory to match at least the best known objects (the Sun and noninteracting binary systems) before any attempt to derive proper information for star clusters or stellar populations in galaxies is made.

Some binary stars are known to provide uncomparable astrophysical laboratories to calibrate theoretical stellar evolutionary models. Since the individual components of welldetached binary systems can be assumed to be two single-like stars with a common origin, they share the same chemical composition and same age, and, therefore, the observed parameters of both components are expected to be matched by a single isochrone at the same chemical composition.

Whilst in general a good overall agreement is found, some studies (pioneered by Popper 1997; see also e.g.

Send offprint requests to: J. Fernandes, e-mail: jmfernan@mat.uc.pt
Pols et al. 1997; Lastennet et al. 1999; Clausen et al. 1999; Lastennet \& Valls-Gabaud 2002 for a systematic analysis) have unambiguously found that systems with components in the 0.7 to $1.1 M_{\odot}$ mass range raise serious difficulties.

There are many well-studied systems with components in this mass range which present puzzling discrepancies with the predictions of stellar evolution theory. For example, if the seismic observations of $\alpha$ Cen A are fitted to state-of-the-art structure models, the resulting masses differ from the ones derived from dynamical analyses (Pourbaix et al. 2002; Thévenin et al. 2002). The visual binary 85 Peg, some eclipsing binaries in the field and in the Hyades open cluster are other clear examples (see Lastennet et al. 2002 for a brief review of the results obtained so far on these objects).

This situation is very puzzling because one would expect these stars to be reasonably well understood. Indeed, stars with masses larger than $\sim 0.6 M_{\odot}$ bypass difficulties in the treatment of the equation of state and the atmosphere, while stars with masses larger than about $1.1 M_{\odot}$ have a permanent convective 
core, introducing an additional parameter, the amount of overshooting, for their modelling. Hence, current stellar evolutionary models are expected to be able to match the basic properties of stars in the $0.7-1.1 M_{\odot}$ mass range, provided a good description of the convection in their envelopes is used.

One of these puzzling objects is the eclipsing binary UV Piscium (HD 7700, HIP 5980, hereafter UV Psc), a detached main-sequence binary (with components of types $\mathrm{G}$ and $\mathrm{K}$ ), which is also a short period ( $<1$ day) $\mathrm{RS} \mathrm{CVn}^{1}$. This binary deserves particular attention ever since Popper (1997) derived age estimates for each component which differed by a factor larger than two, with an absolute age difference larger than 11 Gyr. Montesinos et al. (1988) classified its components as "apparently normal main-sequence stars". What is the origin of this discrepancy?

Popper (1997) used the Geneva models (Schaller et al. 1992) for $Z=0.02$ and $Y=0.30$, i.e. with a helium abundance to metallicity ratio fixed to $\Delta Y / \Delta Z=3$, and $\alpha_{\text {MLT }}$ fixed to a solar-calibrated value $\left(\alpha_{\text {MLT }}=1.63\right)$.

Since the RS Canum Venaticorum stars show activity, mass transfer episodes between active regions of both components are possible. In this case, the application of the single-star theoretical models used by Popper may not be relevant since the evolution of both components would not be independent any longer. However, for UV Psc, mass transfer does not account for the age difference because the computation of the Roche lobe radii (e.g. with the formulae given by Eggleton 1983) shows that none of these stars have overflown their Roche lobes. Therefore, we consider that UV Psc is a well-detached system and assume that the evolution of each component is independent.

Another concern about RS CVn stars is the possible distortion of light curves due to starspots, which could bias the derivation of their fundamental stellar parameters. Jassur \& Kermani (1994) suggested the presence of cool spots on the surface of the primary component, but according to Popper (1997, and references therein), this should not affect the value of the quantities used in this paper. The period has been recently revised by Sowell et al. (2001) who didn't find any evidence for a cyclic modulation or period change. Hence activity cannot account for the age discrepancy either.

To tackle this problem, we explore the influence of each physical parameter. A stellar evolutionary code allowing to compute tracks for different and independent physical parameters is needed for such a purpose, and so we choose the CESAM code (Morel 1997) which fulfills this condition and which has already been successfully tested in a variety of astrophysical contexts (e.g. Zahn et al. 1997; Suran et al. 2001; Cordier et al. 2002).

In this paper, we present a detailed study of the system UV Psc with the CESAM models. Our goal is twofold: 1) to check if there exists a solution giving consistent ages for

\footnotetext{
${ }^{1}$ RS CVn are binary systems where one of the components presents an extreme form of solar-like activity with starspots and variable magnetic fields. See Montesinos et al. (1988) for a discussion.
}

both components for a set of $(Z, Y)$, and 2) to constrain $\alpha_{\mathrm{MLT}}$ for both components and check if these values are consistent with the generally assumed solar value, $\alpha_{\text {MLT } \odot}$.

The paper is organised as follows: Sect. 2 deals with the description of the CESAM models used and the grid computed for the purpose of this paper. Section 3 presents the results which fit both components of the eclipsing binary UV Psc, with particular emphasis on the constraints derived from the $T_{\text {eff }}$-radius diagram on the $\alpha_{\text {MLT }}$ parameters. We also discuss the robustness of the results against changes in the $T_{\text {eff }}$ scale and in diffusion. Finally, Sect. 4 provides a summary of our results.

\section{Evolutionary stellar models}

The stellar evolution calculations were computed with the CESAM code (version 3, Morel 1997), running at the Coimbra Observatory. Details on the physics of these models can be found in Lebreton et al. (1999). Here we just provide a short summary of the main CESAM physical inputs: the CEFF equation of state is used, including Coulomb corrections to the pressure (Eggleton et al. 1973; Christensen-Dalsgaard 1991); the nuclear reactions rates are from Caughlan \& Fowler (1988); the solar mixture is from Grevesse \& Noels (1993); the OPAL opacities (Iglesias \& Rogers 1996) are used and complemented at low temperatures by opacity data from Alexander \& Ferguson (1993) following a prescription by Houdek \& Rogl (1996); the atmosphere is described with an Eddington $T(\tau)$-law; the convection is treated according to the mixing-length theory from Böhm-Vitense (1958), with the formalism of Henyey et al. (1965), giving the mixing-length scale $\left(\alpha_{\mathrm{MLT}} \times H_{\mathrm{p}}\right)$ in terms of a free parameter times the local pressure scale height $H_{\mathrm{p}}$.

With these physical ingredients, we computed a solar model that fits the observed luminosity and radius with an accuracy of $10^{-4}$, with $\alpha_{\mathrm{MLT}}=1.63$, helium abundance $Y_{\odot}=0.268$ and $Z_{\odot}=0.0175$ for the commonly accepted solar age of $\sim 46$ Gyr (Dziembowski et al. 1999) and solar abundances as derived by Grevesse \& Noels (1993).

Given this calibration, we computed a large grid of CESAM models from the Zero Age Main Sequence (ZAMS) to the terminal Age Main Sequence (TAMS, central hydrogen exhaustion), or for ages $t$ younger than $10 \mathrm{Gyr}^{2}$. This was done for each component of UV Psc by fixing the masses to their accurately measured values: $M_{\mathrm{A}}=0.975 M_{\odot}$ and $M_{\mathrm{B}}=0.760$ $M_{\odot}$ (Popper 1997). This approximation is justified since the relative mass accuracy is better than $1 \%$ in each star.

We considered different values of the metallicity $Z(0.01$, $0.02,0.03,0.04$ and 0.05$)$, helium content $Y(0.25,0.28$, 0.31 and 0.34$)$ and mixing length parameter $\alpha_{\text {MLT }}(0.8,1.3$ and 1.7) in a large three-dimensional grid, since we do not want to introduce correlations between these parameters.

\footnotetext{
2 UV Psc being a binary belonging to the close solar neighbourhood $(d \approx 63 \mathrm{pc}$ ), ages older than the Galactic disc (likely to be 8-10 Gyr old, at most, see Carraro 2000) should be irrelevant here.
} 


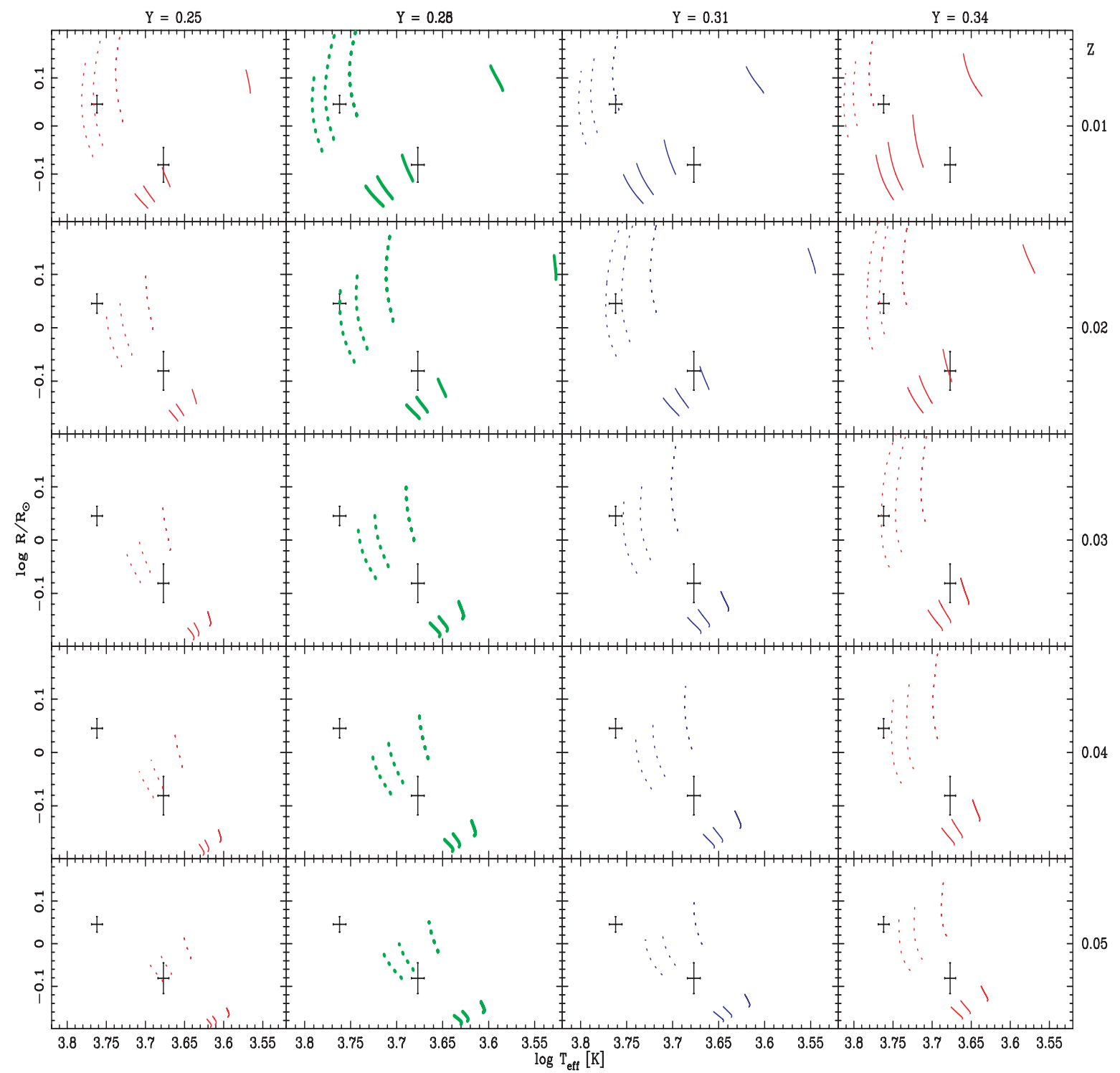

Fig. 1. Location of UV Psc A and B (error bars in bold, the primary component being the hottest one) in the effective temperature-radius diagram with evolutionary tracks computed from the CESAM code for various chemical compositions. On each panel, tracks with the mass of the primary (dashed lines) and the secondary (solid lines) components are shown for 3 different values of $\alpha_{\mathrm{MLT}}: \alpha_{\mathrm{MLT}}=1.7,1.2$ and 0.8 (from left to right). For $Z=0.01$ and $Z=0.02$, we also show $\alpha_{\mathrm{MLT}}=0.1$ (solid line at the coolest temperatures) for the secondary component. All tracks are computed from the ZAMS to the TAMS or to $10 \mathrm{Gyr}$ (see text for details).

We also computed additional models for the secondary component with $\alpha_{\mathrm{MLT}}=0.1$ for $Z=0.01$ and $Z=0.02^{3}$. We stress that the quantities $Z$ and $Y$ are independent, unlike most of the extant publicly available theoretical models where $Y$ is derived from a fixed law once $Z$ is determined. All the models computed with the input physics described above are displayed in Fig. 1.

\footnotetext{
${ }^{3}$ The internal structure of such low- $\alpha_{\mathrm{MLT}}$ models does not present any convective region at all (this can be explained by the fact that the convective efficiency is proportional to the square of the mixing length and hence to $\alpha_{\mathrm{MLT}}^{2}$ ). The discussion on how realistic these models are is beyond the scope of this paper, but we would like to point out that we only used them to interpolate models with $\alpha_{\text {MLT }}$ slightly lower than 0.8 .
}

\section{Analysis of the results}

UV Psc is an eclipsing binary with very accurate masses and radii derived for both components. The fundamental data used in this paper (from Popper 1997) are listed in Table 1 and are a key point of this study: the measures by Popper represent such a significant revision and improvement that a comparison with older data is irrelevant.

For instance, Montesinos et al. (1988) provided a comprehensive work on RS CVn EBs and derived a metal-rich solution for UV Psc $(Z=0.04$ and $Y=0.25)$ from the $\log g-T_{\text {eff }}$ diagram, but the data they used were very significantly revised by Popper $\left(\Delta M_{A}=0.3 M_{\odot}\right.$, etc. $)$ hampering any useful comparison.

First, before exploring all possible solutions in the $(Z, Y$, $\left.\alpha_{\mathrm{MLT}}, t\right)$ parameter space, interesting qualitative conclusions 
Table 1. Parameters from Popper (1997) for the primary (A) and secondary (B) components of UV Psc.

\begin{tabular}{cccc}
\hline \hline Comp. & $M$ & $R$ & $\log \left(T_{\text {eff }} / \mathrm{K}\right)$ \\
& {$\left[M_{\odot}\right]$} & {$\left[R_{\odot}\right]$} & \\
\hline $\mathrm{A}$ & $0.975 \pm 0.009$ & $1.11 \pm 0.02$ & $3.762 \pm 0.007$ \\
$\mathrm{~B}$ & $0.760 \pm 0.005$ & $0.83 \pm 0.03$ & $3.677 \pm 0.007$ \\
\hline
\end{tabular}

can be derived from a simple inspection of Fig. 1. Whatever the values of $Y$ and $\alpha_{\text {MLT }}$ are, all models with $Z \geq 0.04$ are unable to match simultaneously the location of both components, strongly suggesting that metal-rich solutions can be ruled out. In addition, while a fit to both components seems to be possible in some panels -e.g., the upper left panel at $(Z, Y)=(0.01$, 0.25 )- inconsistent ages appear, excluding these possible solutions. This is also the case for the solution found by Popper at $(Z, Y)=(0.02,0.30)$ using the Geneva models.

Altogether, this means that we expect to find solutions with consistent ages for chemical compositions in the range $Z=$ $0.01-0.02$ and $Y=0.28-0.34$. At this level of qualitative discussion, it appears difficult to infer strong conclusions about the $\alpha_{\text {MLT }}$ parameter but the panels of Fig. 1 suggest that $\alpha_{\text {MLT,B }}$ may be systematically smaller that $\alpha_{\text {MLT,A }}$.

To obtain more detailed conclusions, a systematic work matching some selected relevant criteria has to be performed. For this purpose, we explore by interpolation ${ }^{4}$ the $\left(Z, Y, \alpha_{\mathrm{MLT}}\right.$, $t$ ) parameter space described in the previous section to find out the best mathematical solutions matching the location of UV

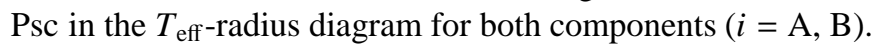
This is performed by minimizing the $\chi^{2}$-functional

$$
\begin{aligned}
\chi^{2}\left(Z_{i}, Y_{i}, \alpha_{\mathrm{MLT} i}, t_{i}\right)= & \left(\frac{\log T_{\mathrm{eff}}(\operatorname{track})-\log T_{\mathrm{eff} i}}{\sigma\left(\log T_{\mathrm{eff} i}\right)}\right)^{2} \\
& +\left(\frac{\log R(\operatorname{track})-\log R_{i}}{\sigma\left(\log R_{i}\right)}\right)^{2} .
\end{aligned}
$$

We assume that both components share the same chemical composition, i.e. $Z_{\mathrm{A}}=Z_{\mathrm{B}}$ and $Y_{\mathrm{A}}=Y_{\mathrm{B}}$. Moreover, they should also have equal (or at least consistent) ages, hence $\left\|t_{\mathrm{A}}-t_{\mathrm{B}}\right\|$ should be minimal.

In Fig. 2, we show all the solutions in three projections: $Z-Y, Z-\alpha_{\mathrm{MLT}, \mathrm{A}}$ and $Z-\alpha_{\mathrm{MLT}, \mathrm{B}}$ diagrams. The solutions (small dots in Fig. 2) which have a good $\chi^{2}$ for both components $\left(\chi_{i}^{2} \leq\right.$ $\chi_{i, \text { min }}^{2}+1$ with $\left.i=\mathrm{A}, \mathrm{B}\right)$ show that i) high metallicities $(Z>$ 0.032 ) are indeed ruled out, ii) $1.7 \geq \alpha_{\mathrm{MLT}, \mathrm{A}} \geq 0.9$, and iii) $1.0 \geq$ $\alpha_{\text {MLT,B }} \geq 0.6$.

Adding the constraint that the ages are consistent with each other $(\Delta t / t \leq 20 \%)$ provides the subsample shown with crosses. It defines a much smaller region (Fig. 2) and shows that i) the metallicity is solar or sub-solar $(Z<0.019)$ and the helium content is larger than the solar value $(Y>0.28)$, ii) $1.1 \geq \alpha_{\text {MLT,A }} \geq$ 0.9 , and iii) $0.7 \geq \alpha_{\mathrm{MLT}, \mathrm{B}} \geq 0.6$.

The best solution (filled circle in Fig. 2) is obtained with a $Z=0.012, Y=0.31$ chemical composition and provides an excellent agreement in age ( $0.9 \%$ discrepancy). We predict

\footnotetext{
${ }^{4} \mathrm{Z}, Y$ and $\alpha_{\mathrm{MLT}}$ in steps of $0.001,0.005$ and 0.05 , respectively.
}

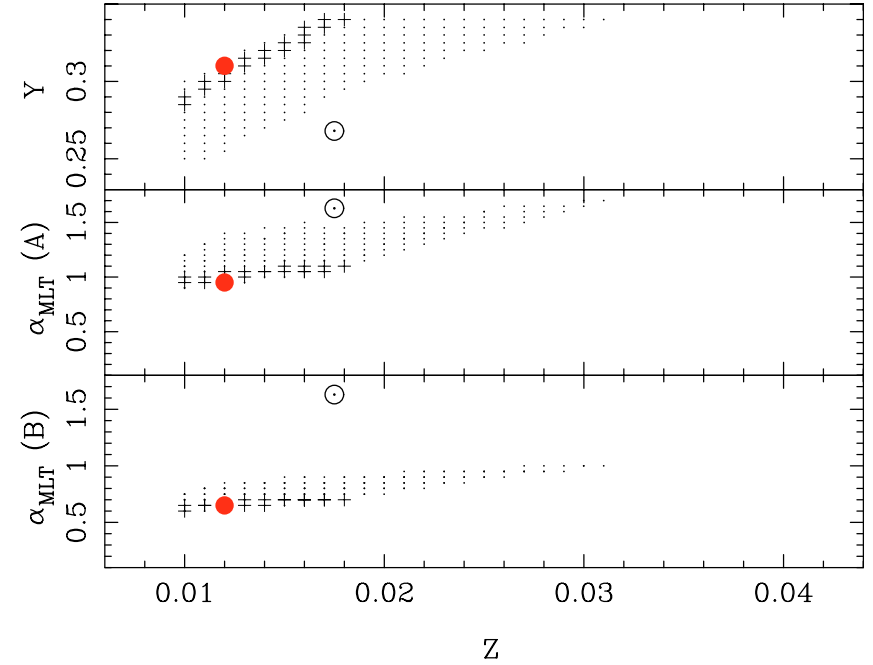

Fig. 2. $\left(Z, Y, \alpha_{\mathrm{MLT}, \mathrm{A}}, \alpha_{\mathrm{MLT}, \mathrm{B}}\right)$ solutions (dots) matching the location of both UV Psc A and B in the $T_{\text {eff }}$-radius diagram ( $T_{\text {eff }} \mathrm{s}$ from Popper 1997 ) with the CESAM evolutionary tracks. Adding a constraint to obtain consistent ages for both components $(\Delta t / t \leq 20 \%)$ provides the subsample shown as crosses. The best agreement in age $(0.9 \%)$ is shown with a filled circle. The Sun (dotted circle) is also shown for comparison.

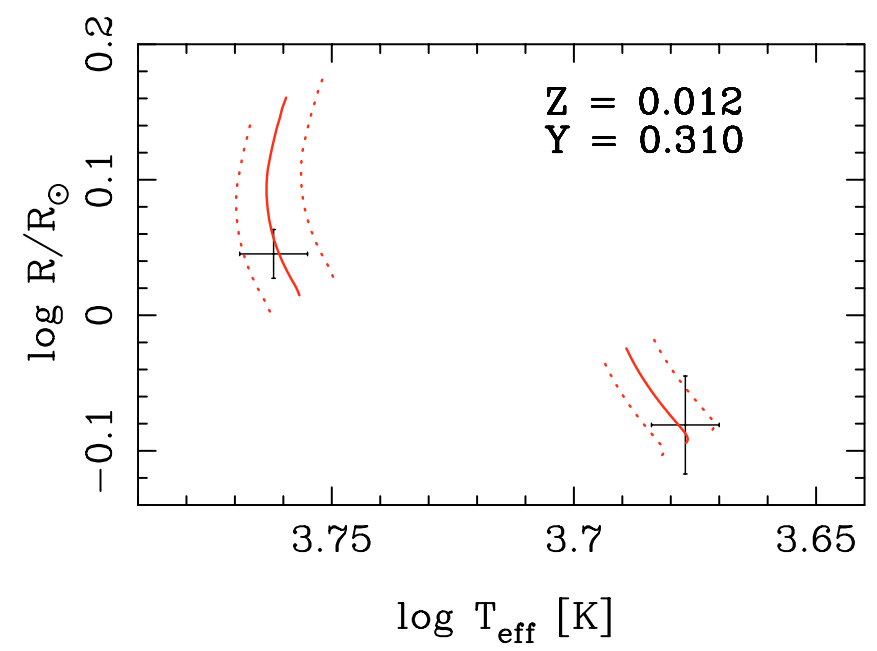

Fig. 3. Best fit solution (filled circle in Fig. 2) for both UV Psc A and $\mathrm{B}$ in the temperature-radius diagram $\left(T_{\mathrm{eff}} \mathrm{S}\right.$ and radii from Popper $1997)$ with the evolutionary tracks computed from the CESAM code: $\alpha_{\mathrm{MLT}, \mathrm{A}}=0.95 \pm 0.12$ and $\alpha_{\mathrm{MLT}, \mathrm{B}}=0.65 \pm 0.07$.

an age of about 1.9 Gyr for both components of UV Psc A $\left(1.92_{-0.4}^{+0.6} \mathrm{Gyr}\right)$ and B $\left(1.94_{-0.4}^{+3.0} \mathrm{Gyr}\right)$. While a unique $(Z, Y, t)$ combination is needed to simultaneously match the (masses, radii, $T_{\text {eff }} \mathrm{s}$ ) of both components, this solution implies a different value for the convection parameter for the two components of the binary: $\alpha_{\mathrm{MLT}}(\mathrm{UV}$ Psc A) $=0.95 \pm 0.12$ and $\alpha_{\mathrm{MLT}}(\mathrm{UV}$ Psc B) $=0.65 \pm 0.07$. The fit provided by this solution is shown in Fig. 3.

The non-solar values for $\alpha_{\text {MLT }}$ parameters that we derive obviously deserve further comments, but the robustness of these solutions needs to be discussed first. 


\subsection{Influence of the $T_{\text {eff }}$ scaling}

Since our main concern was to solve the age discrepancy found by Popper (1997), we used the same values for the mass, radius and $T_{\text {eff }}$. We have shown in the previous section that this discrepancy can be solved within the same data set. However, whilst the individual masses and radii are very accurately defined, the $T_{\text {eff }}$ s are not direct quantities, and depend on colour indices and photometric calibrations. We would like to point out that according Popper (1998) the presence of dark spots has relatively little effect on the color index of a star. To test the possible influence of the $T_{\text {eff }}$ scaling on our results, we used as an alternative the metallicity-dependent empirical calibrations of Alonso et al. (1996), $T_{\text {eff }}=f(B-V,[\mathrm{Fe} / \mathrm{H}])$ and used the $B-V$ indices of both components from Table 6 of Popper (1997) assuming that the reddening can be neglected because UV Psc is in the inner solar neighbourhood ( $\sim 63 \mathrm{pc}$ according to its Hipparcos parallax). Since there is no direct determination of the iron abundance $[\mathrm{Fe} / \mathrm{H}]$ available for UV Psc, we have computed both $T_{\text {eff }}$ s from the Alonso et al.'s formula (1) assuming three different metallicities: $T_{\text {eff, }}=5574 \mathrm{~K}$ and $T_{\text {eff, }, \mathrm{B}}=4506 \mathrm{~K}$ $(Z=0.01), T_{\text {eff }, \mathrm{A}}=5682 \mathrm{~K}$ and $T_{\text {eff }, \mathrm{B}}=4586 \mathrm{~K}(Z=0.02)$, $T_{\text {eff,A }}=5754 \mathrm{~K}$ and $T_{\text {eff, } \mathrm{B}}=4638 \mathrm{~K}(Z=0.03)$. The uncertainty on the primary (secondary) component is less than $150 \mathrm{~K}$ $(100 \mathrm{~K})$, and so - to be conservative - we consider these upper limits as the uncertainty. For further comparisons, we take the $T_{\text {eff }} \mathrm{s}$ derived for $Z=0.02$. They are cooler by about $100 \mathrm{~K}$ $(170 \mathrm{~K})$ for the primary (secondary) component in comparison to the $T_{\text {eff }}$ s that we have used previously (see Table 1 ).

The influence of adopting the $T_{\mathrm{eff}} \mathrm{S}$ we have derived from the Alonso et al. calibrations can be seen in Fig. 4. A comparison with Fig. 2 shows that the global solutions (dots) are fully consistent with each other. However, the best solution according to the criteria defined in Sect. 3 is slightly different: $(Z, Y)=(0.015,0.295)$, the ages predicted being about twice older (UV Psc A: 4.07 Gyr and B: 4.14 Gyr). In spite of these shifts, we note that a different value is still needed for the convection parameter of the two components $\left(\alpha_{\mathrm{MLT}, \mathrm{A}}=1.15\right.$ and $\left.\alpha_{\mathrm{MLT}, \mathrm{B}}=0.65\right)$. The difference $\Delta \alpha_{\mathrm{MLT}}$ is even increased in comparison to the results inferred from the Popper (1997)'s $T_{\text {eff }}$ scale, and hence the conclusions of the previous sub-section remain qualitatively unchanged. Quantitatively, a systematic error of about +0.20 in $\alpha_{\text {MLT,A }}$ (and a negligible one in $\alpha_{\text {MLT,B }}$ ) may be due to the uncertainties in the temperature scaling.

\subsection{Influence of diffusion}

Another potential source of systematic error is the influence of diffusion. We have treated this by computing CESAM models incorporating the microscopic diffusion as described by the simplified formalism proposed by Michaud \& Proffitt (1993) with metal elements as trace elements. Note that the radiative acceleration can be neglected for sub-solar stars (Turcotte et al. 1998). As shown in Fig. 5, diffusion almost mimics an increase in $\alpha_{\mathrm{MLT}}$, even though the slopes of the tracks are obviously changed. At fixed radius, the effect of diffusion is to change the effective temperatures by some $65 \mathrm{~K}(55 \mathrm{~K})$, well within the 1- $\sigma$ uncertainties in $T_{\text {eff }}$ discussed above. Alternatively, taking

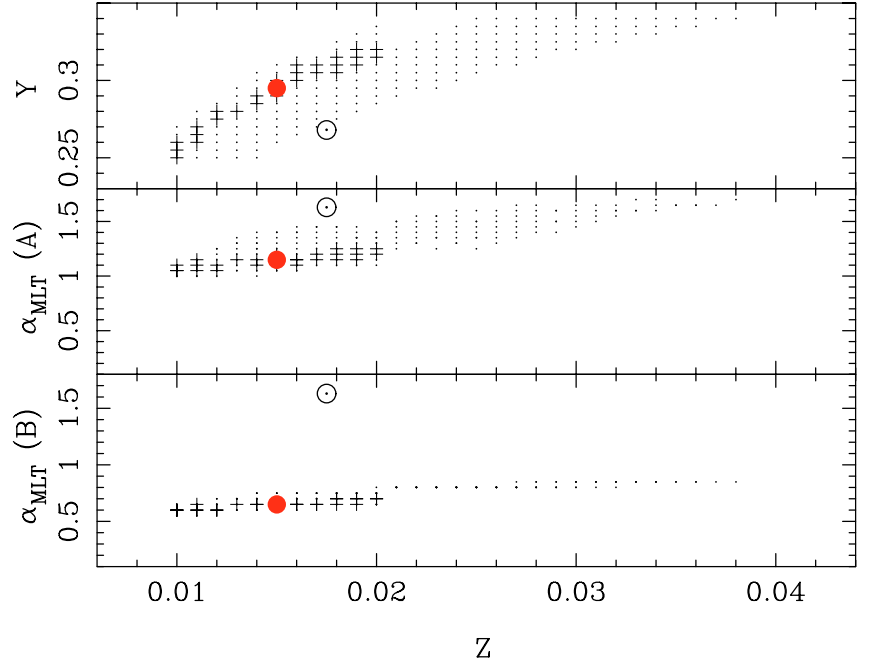

Fig. 4. Same as Fig. 2 but with the $T_{\text {eff }} \mathrm{s}$ derived from the Alonso et al. (1996) calibrations. The best agreement in age (1.6\%) is shown as a filled circle.

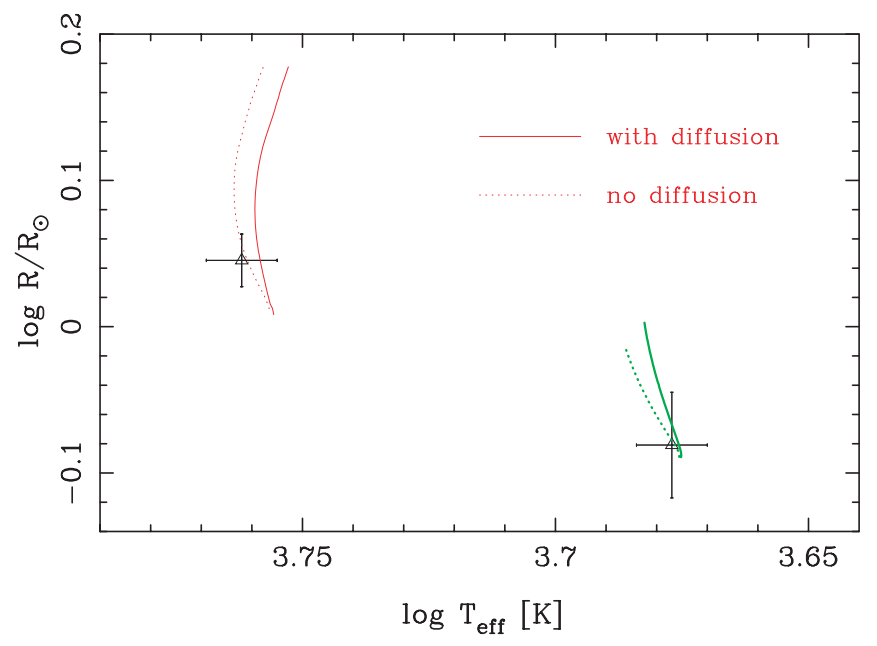

Fig. 5. The effect of including microscopic diffusion models, assuming the same composition $(Z, Y)=(0.012,0.31)$ for each component.

these uncertainties at face value, one would have to increase $\alpha_{\text {MLT }}$ by about $0.11(0.07)$ at most.

In summary, even though the absolute values of $\alpha_{\text {MLT }}$ may change due to these combined effects, the trend of increased $\alpha_{\text {MLT }}$ with mass remains robust.

\subsection{Universality of the $\alpha_{M L T}$ parameter ?}

We have quantitatively confirmed the suggestion made by Clausen et al. (1999) that the adoption of a significantly smaller $\alpha_{\mathrm{MLT}}$ for the secondary component may remove (or at least decrease) the general problem of age discrepancy presented in the Introduction. While it is obvious that only observational constraints on the chemical composition of UV Psc would allow a proper test, it seems that this problem can be solved theoretically for this binary. The solution obtained for this system suggests that i) the components have a different $\alpha_{\mathrm{MLT}}$ $\left(\Delta \alpha_{\mathrm{MLT}}=0.3 \pm 0.14\right)$ and ii) these values are clearly different from the solar one. 


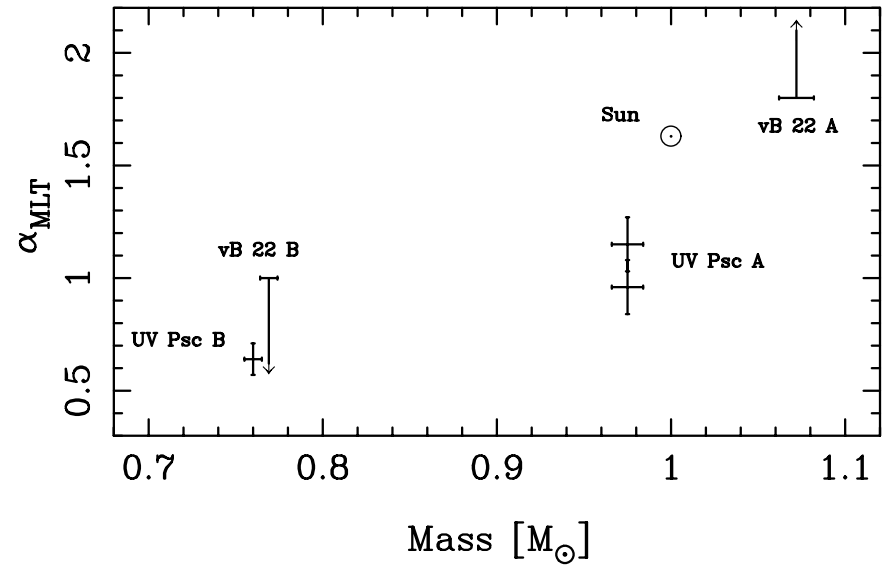

Fig. 6. Best fit solution for both UV Psc A and B in a mass- $\alpha_{\mathrm{MLT}}$ diagram. The value of $\alpha_{\mathrm{MLT}, \mathrm{A}}$ is sligthly different according to the assumed $T_{\text {eff }} \mathrm{s}: \alpha_{\mathrm{MLT}}=0.95$ (Popper 1997), $\alpha_{\mathrm{MLT}}=1.15$ (Alonso et al. 1996, see text). The solution is independent of the choice of the $T_{\text {eff }} \mathrm{s}$ for the secondary component. The Sun (cf. Sect. 2) is shown for comparison, as well as estimates (shown as upper and lower limits) derived from CESAM models with the same input physics for both components of the Hyades binary vB 22 (Lebreton et al. 2001).

This implies that the solar value of the $\alpha_{\text {MLT }}$ is not universal, an hypothesis made in most of the currently available stellar evolutionary models.

As for the trend with mass, a recent study on the Hyades eclipsing binary vB22 (Lebreton et al. 2001) seems to support it. We show in Fig. 6 that UV Psc and vB22 are clearly not consistent with the solar $\alpha_{\text {MLT }}$ value. Moreover, Lebreton et al. (2001) show that the slope of the main-sequence in the Hyades suggests that $\alpha_{\text {MLT }}$ could decrease with mass, in particular they obtained $\alpha_{\text {MLT }} \leq 1.4$ below $1 M_{\odot}$. The results we have obtained for UV Psc give additional support to this trend ${ }^{5}$. However we would like to point out that this trend seems to contradict the results of Ludwig et al. (1999) coming from detailed hydrodynamical simulations where, for a fixed gravity, $\alpha_{\mathrm{MLT}}$ increases with deacreasing $T_{\text {eff }}$.

More results are needed before reaching any definitive conclusion, because a larger sample may well reveal a scatter of $\alpha_{\mathrm{MLT}}$ for a given mass. For instance, recent calibrations of $\alpha_{\mathrm{MLT}}$ in visual binary stars were performed by P. Morel and collaborators using the CESAM code (with different physical ingredients than those used in this paper). For the stars in the range of mass relevant in this paper, they find $\alpha$ Cen $\mathrm{B}, M=0.97 M_{\odot}, \alpha_{\mathrm{MLT}}=1.53$ (Morel et al. 2000a), $\iota \mathrm{Peg}$ $\mathrm{B}, M=0.81 M_{\odot}, \alpha_{\mathrm{MLT}}=1.36$ (Morel et al. 2000b) and 85 Peg A, $M=0.84 M_{\odot}, \alpha_{\mathrm{MLT}}=1.80$ (Fernandes et al. 2002). Since both $\iota$ Peg B and 85 Peg A have very similar masses, this seems to support the idea of a dispersion of $\alpha_{\text {MLT }}$ at a given mass. Solar models with and without diffusion yield different values in the internal convective regions (1.8 vs. 2.0,

\footnotetext{
${ }^{5}$ Iwamoto \& Saio (1999) also give a trend of $\alpha_{\mathrm{MLT}}$ increasing with mass and metallicity but their results are for giant stars, where overshooting is playing a major role, so disentangling their relative effects is not trivial.
}

Morel et al. 1997). At larger masses, $\alpha_{\text {MLT }}$ seems to increase to values up to 3 to reproduce the red giant branches in open clusters (Stothers \& Chin 1997). Therefore, at this stage, a relation between mass and $\alpha_{\mathrm{MLT}}$ is highly premature and must be seen with caution.

We also note, in passing, that our results are not in contradiction with the recent analysis by Palmieri et al. (2002), who on the basis of the colours of red giants in globular clusters concluded that there is no dependence of $\alpha_{\mathrm{MLT}}$ with metallicity. The extremely narrow range in mass probed by these red giants would be nearly equivalent to a single point on our diagram. Since the detailed modelling of red giants is more complicated than the one of main sequence stars, a larger sample of double-lined, eclipsing binaries with components in the 0.7-1.1 $M_{\odot}$ would offer a much cleaner and definitive test.

In any case, all these possible variations and their trends reflect a shortcoming of the MLT theory more than a real physical effect. If one insists in using MLT, its parameter $\alpha_{\text {MLT }}$ should be varied. This is perhaps the first time that such a result is obtained using observations, since the variablity of the effective $\alpha_{\text {MLT }}$ is a well-know result of detailed hydrodynamical simulations of stellar convection (Ludwig et al. 1999).

On the other hand we would like to note that the value of $\alpha_{\text {MLT }}$ determined in atmosphere of the stars gives in general different results in relation to those obtained from the stellar interior analysis. For instance, van't Veer-Menneret \& Mégessier (1996) found that the spectral fitting of the first for Balmer lines of the solar spectrum required $\alpha_{\mathrm{MLT}}=0.5$, a result confirmed by Fuhrmann et al. (1993) for other cool stars, while Gardiner et al. (1999) found that $\alpha_{\mathrm{MLT}} \sim 0.5$ for $T_{\mathrm{eff}} \mathrm{s}$ below $6000 \mathrm{~K}$ but $\alpha_{\mathrm{MLT}} \geq 1.25$ in the range $6000-7000 \mathrm{~K}$ to possibly decrease again to $\alpha_{\mathrm{MLT}}=0.5$ above $7000 \mathrm{~K}$.

\subsection{Implications for the helium to metal ratio}

Since we have derived $Z$ and $Y$ independently for UV Psc, the helium to metal ratio $(\Delta Y / \Delta Z)$ of this system can be inferred assuming a primordial helium abundance. The initial helium content is fixed by taking the currently assumed law $Y=Y_{\mathrm{p}}+$ $(\Delta Y / \Delta Z) \times Z$, where $Y_{\mathrm{p}}$ is the primordial helium abundance. If we consider $Y_{\mathrm{p}}=0.235$ (Peimbert et al. 2000) and use the $(Z$, $Y$ ) solution that we have found for the UV Psc binary, we derive $\Delta Y / \Delta Z \sim 6$ and 4 by using the Popper (1997) or Alonso et al. (1996) $T_{\text {eff }} \mathrm{s}$, respectively. In both cases, the ratio is large in comparison to what can be obtained from the solar model described previously: $(\Delta Y / \Delta Z)_{\odot} \sim 1.9$. This may suggest that the currently assumed simple linear relationship between $Z$ and $Y$ is not universal and/or that $\Delta Y / \Delta Z$ is not unique.

The possible relation between $Y$ and $Z$ is one of the most interesting and puzzling problems in astrophysics since the pioneering work by Faulkner (1967).

The most recent observations in HII regions and planetary nebulae as well as semi-empical determinations using theoretical stellar models - including the detailed calibration of the Sun - (cf. Ribas et al. 2000; Fernandes 2001 and references therein) support values around 2 or 3 , assuming $Y_{\mathrm{p}} \sim 0.23$. 
Nevertheless, some exceptions indicate values larger than 4 (e.g. Pagel et al. 1992 from observations of extragalactic HII regions, and Belikov et al. 1998 from the main sequence of the Pleiades). Our determination clearly supports the idea of a large $\Delta Y / \Delta Z$ ratio.

\section{Conclusions}

We study the eclipsing binary UV Psc, which is of particular interest since Popper (1997) derived age estimates for both components which differed by some 11 Gyr. Adopting his same data set (masses, radii and $T_{\text {eff }} \mathrm{s}$ ) we compute a large grid of CESAM models for each component ${ }^{6}$. We obtain a best fit in the $T_{\text {eff }}$-radius diagram for a common chemical composition $(Z, Y)=(0.012,0.31)$, a negligible age difference, and a different MLT parameter $\left(\alpha_{\mathrm{MLT}, \mathrm{A}}=0.95 \pm 0.12\right.$ and $\left.\alpha_{\mathrm{MLT}, \mathrm{B}}=0.65 \pm 0.07\right)$. The difference $\alpha_{\mathrm{MLT}, \mathrm{A}}-\alpha_{\mathrm{MLT}, \mathrm{B}}=0.3$ is significant and remains robust against changes in temperature calibrations and the inclusion of diffusion.

Within the framework of the MLT theory, this suggests that fixing $\alpha_{\text {MLT }}$ to its solar value ( 1.6), the usual hypothesis made by most stellar evolutionary models, may not be correct. Secondly, since the $\alpha_{\mathrm{MLT}}$ is smaller for the less massive component, this suggests that the $\alpha_{\text {MLT }}$ parameter may decrease with the stellar mass. This trend needs further confirmation with other binary stars with accurate data. Again, this may reflect a problem of the application of the MLT theory, more than a real physical effect, but it has to be taken into account when using MLT. On the other hand as the main observational errors come from the $T_{\text {eff }}$, new and independent determinations are needed for both stars.

So far, few other binaries have data with the precision required to confirm this trend and observational programs are highly needed (e.g. Kurpinska-Winiarska \& Oblak 2000).

Acknowledgements. We warmly thank P. Morel for making available CESAM, a superb tool for stellar evolution, and without which this study could not have been performed. EL is supported by a "Fundação para a Ciência e Tecnologia" (FCT) postdoctoral fellowship (grant SFRH/BPD/5556/2001). This work was partially supported by the project "PESO/P/PRO/15128/1999" from the FCT, and by "Convénio Embaixada de França-ICCTI (59-B0)". This research has made use of the SIMBAD database operated at CDS, Strasbourg, France, and of NASA's Astrophysics Data System Abstract Service.

\section{References}

Alexander, D. R., \& Ferguson, J. W. 1993, ApJ, 437, 879

Alonso, A., Arribas, S., \& Martinez-Roger, C. 1996, A\&A, 313, 873

Barklem, P. S., Stempels, H. C., Allende Prieto, C., et al. 2002, A\&A, 385,951

\footnotetext{
6 These models are available upon request (e-mail: jmfernan@mat.uc.pt).
}

Belikov, A. N., Hirte, S., Meusinger, H., Piskunov, A. E., \& Schilbach, E. 1998, A\&A, 332, 575

Böhm-Vitense, E. 1958, Zs. Ap 46, 108

Carraro, G. 2000, The Evolution of the Milky Way: stars versus clusters, ed. F. Matteucci, \& F. Giovannelli (Kluwer Academic Publishers), 335

Caughlan, G. R., \& Fowler, W. A. 1988, Atomic Data Nuc. Data Tables, 40, 284

Clausen, J. V., Baraffe, I., Claret, A., \& VandenBerg, D. A. 1999, ASP Conf. Ser., 173, 265

Christensen-Dalsgaard, J. 1991, in Challenges to theories of the structure of moderate-mass stars, ed. D. O. Gough, \& J. Toomre (Springer-Verlag), 11

Cordier, D., Lebreton, Y., Goupil, M.-J., Lejeune, T., et al. 2002, A\&A, 392, 169

Dziembowski, W. A., Fiorentini, G., Ricci, B., \& Sienkiewicz, R. 1999, A\&A, 343, 990

Eggleton, P. P. 1983, ApJ, 268, 368

Eggleton, P. P., Faulkner, J., \& Flannery, B. P. 1973, A\&A, 23, 325

Faulkner, J. 1967, ApJ, 147, 617

Fernandes, J. 2001, Proc. of the 10th Portuguese Meet., ed. J. P. S. Lemos, A. M. Mourao, L. Teodoro, \& R. Ugoccioni (World Scientific), 149

Fernandes, J., Morel, P., \& Lebreton, Y. 2002, A\&A, 392, 529

Fuhrmann, K., Axer, M., \& Gehren, T. 1993, A\&A, 271, 451

Gardiner, R. B., Kupka, F., \& Smalley, B. 1999, A\&A, 347, 876

Grevesse, N., \& Noels, A. 1993, in Origin and Evolution of the Elements, ed. N. Prantzos, E. Vangioni-Flam, \& M. Cassé (Cambridge University Press)

Henyey, L., Vardya, M. S., \& Bodenheimer, P. 1965, ApJ, 142, 841

Houdek, G., \& Rogl, J. 1996, Bull. Astron. Soc. Ind., 24, 317

Iglesias, C. A., \& Rogers, F. J. 1996, ApJ, 464, 943

Iwamoto, N., \& Saio, H. 1999, ApJ, 521, 297

Jassur, D. M. Z., \& Kermani, M. H. 1994, A\&AS, 219, 35

Kurpinska-Winiarska, M., \& Oblak, E. 2000, IAU Symp. 200, ed. B. Reipurth, \& H. Zinnecker, 141

Lastennet, E., Fernandes, J., Oblak, E., \& Valls-Gabaud, D. 2002, ed. T. Lejeune, \& J. Fernandes, ASP Conf. Ser., 274, 214

Lastennet, E., \& Valls-Gabaud, D. 2002, A\&A, 396, 551

Lastennet, E., Valls-Gabaud, D., Lejeune, T., \& Oblak, E. 1999, A\&A, 349,485

Lebreton, Y., Fernandes, J., \& Lejeune, T. 2001, A\&A, 374, 540

Lebreton, Y., Perrin, M.-N., Cayrel, R., Baglin, A., \& Fernandes, J. 1999, A\&A, 350, 587

Ludwig, H. G., Freytag, F., \& Steffen, M. 1999, A\&A, 346, 111

Michaud, G., \& Proffitt, C. R. 1993, in Inside the stars, IAU Coll. 137, 246

Montesinos, B., Giménez, A., \& Fernández-Figueroa, M. J. 1988, MNRAS, 232, 361

Morel, P. 1997, A\&AS, 124, 597

Morel, P., Provost, J., \& Berthomieu, G. 1997, A\&A, 327, 349

Morel, P., Morel, Ch., Provost, J., \& Berthomieu, G. 2000a, A\&A, 354,636

Morel, P., Provost, J., Lebreton, Y., Thévenin, F., et al. 2000b, A\&A, 363,675

Pagel, B. E. J., Simonson, E. A., Terlevich, R. T., \& Edmunds, M. G. 1992, MNRAS, 255, 325

Palmieri, R., Piotto, G., Saviane, I., Girardi, L., \& Castellani, U. 2002, A\&A, 392, 115

Peimbert, M., Peimbert, A., \& Ruiz, M. 2000, ApJ, 541, 688

Pols, O. R., Tout, C. A., Schröder, K.-P., Eggleton, P. P., \& Manners, J. 1997, MNRAS, 289, 869 
Popper, D. 1997, AJ, 114, 1195

Popper, D. 1998, PASP, 110, 919

Pourbaix, D., Nidever, D., McCarthy, C., et al. 2002, A\&A, 386, 280

Ribas, I., Jordi, C., Torra, J., \& Giménez, A. 2000, MNRAS, 313, 99

Schaller, G., Schaerer, D., Meynet, G., \& Maeder, A. 1992, A\&AS, 96,269

Sowell, J. R., Hughes, S. B., Hall, D. S., \& Howard, B. A. 2001, AJ, 122,1965
Stothers, R. B., \& Chin, C. 1997, ApJ, 478, L103

Suran, M., Goupil, M., Baglin, A., Lebreton, Y., \& Catala, C. 2001, A\&A, 372, 233

Thévenin, F., Provost, J., Morel, P., et al. 2002, A\&A, 392, L9

Turcotte, S., Richer, J., Michaud, G., Iglesias, C. A., \& Rogers, F. J. 1998, ApJ, 504, 539

van't Veer-Menneret, C., \& Mégessier, C. 1996, A\&A, 309, 879

Zahn, J.-P., Talon, S., \& Matias, J. 1997, A\&A, 322, 320 